\title{
Cosmopolitismo, patriotismo y ecología
}

\author{
Cosmopolitanism, Patriotism, and Ecology
}

\section{Le Cosmopolitisme, le patriotisme, et l'écologie}

\author{
Nel Noddings \\ Stanford University, Estados Unidos
}

\begin{abstract}
RESUMEN
La creciente globalización trae consigo una necesidad cada vez mayor de cooperación internacional y la posibilidad de un renovado interés en el cosmopolitismo. La enseñanza para - incluso acerca de-el cosmopolitismo ocasiona problemas para los docentes en todo el mundo, pero es particularmente difícil para los profesores en Estados Unidos debido al actual resurgimiento del orgullo por el "excepcionalismo estadounidense." Tras una breve discusión de los posibles beneficios y riesgos de enseñar el cosmopolitismo, exploraré un enfoque que puede disminuir los riesgos y aumentar los beneficios. Este enfoque esperanzador podría denominarse "cosmopolitismo ecológico" (Noddings, 2012).
\end{abstract}

Descriptores: Cosmopolitismo y la enseñanza; excepcionalismo; patriotismo; globalización; cosmopolitismo ecológico.

\section{ABSTRACT}

Increasing globalization brings with it an increased need for international cooperation and the possibility of renewed interest in cosmopolitanism. Teaching for - even about - cosmopolitanism raises problems for teachers all over the world, but it is especially difficult for teachers in the United States because of the current revival of pride in "American exceptionalism." After a brief discussion of the possible benefits and risks in teaching cosmopolitanism, I will explore an approach that may decrease the risks and increase the benefits. This promising approach might be called "ecological cosmopolitanism" (Noddings, 2012).

Key words: cosmopolitanism and teaching; exceptionalism; patriotism; globalization; ecological cosmopolitanism.

RÉSUMÉ

La globalisation croissante apporte avec elle un besoin accru de coopération internationale et la possibilité d'un intérêt renouvelé pour le cosmopolitisme. L'enseignement en vue-et même au sujet — du cosmopolitisme suscite des problèmes pour les enseignants à travers le 
monde, mais il est particulièrement difficile pour les enseignants des États-Unis à cause de la renaissance actuelle de la fierté au sujet de «l'exceptionnalisme. » Après une brève discussion au sujet des bienfaits et des risques d'enseigner le cosmopolitisme, l'auteur explore une approche qui peut en diminuer les risques et en augmenter les bienfaits. Cette approche prometteuse pourrait bien être appelée le " cosmopolitisme écologique. " (Noddings, 2012)

Mots clés : Le cosmopolitisme et l'enseignement; l'exceptionnalisme; le patriotisme; la globalisation; le cosmopolitisme écologique.

\section{La idea del cosmopolitismo}

$\mathrm{C}^{\mathrm{L}}$ cosmopolitismo, la idea de pertenecer al mundo entero más que a una nación, tribu o localidad, es tan vieja como los antiguos griegos, pero siempre ha estado nublada por la idea mucho más emocionante del patriotismo nacional. No existen uniformes, banderas, desfiles, música marcial, juramentos públicos o simulacros de batallas para celebrar el cosmopolitismo. En tiempos de paz y prosperidad, admiramos a los "cosmopolitas" - personas altamente cultas que se sienten "en casa" en cualquier parte del mundo. Pero en épocas de conflicto nacional o grupal, es probable que al cosmopolita comprometido se le critique. En tales épocas, "Mi país para bien o para mal" se convierte en el lema orgulloso.

La experiencia de Thomas Paine es instructiva. A pesar de haber defendido la no violencia toda su vida, apoyó firmemente la Revolución estadounidense en el nombre de la justicia social e incluso criticó a sus colegas cuáqueros por rehusarse a portar armas (True, 1995, p.11-15) Los escolares estadounidenses oyen hablar del influyente panfleto de Paine, El Sentido Común, pero es poco probable que hayan oído hablar de su lema cosmopolita "Mi país es el mundo; hacer el bien es mi religión," o de cualquier otra cosa que haya escrito en La Edad de la Razón. Y probablemente no se enterarán que años más tarde, Theodore Roosevelt se refirió a Paine como "un inmundo pequeño ateo" (True, 1995, p. 14).

La historia de vida y esfuerzos de Paine es importante para los docentes cuando tratan de estimular el pensamiento crítico sobre el cosmopolitismo. A pesar de toda una vida de enemistad hacia la violencia, esclavitud e injusticia, Paine fue llamado "un Judas, reptil, cerdo, perro rabioso, borracho, piojo, archienemigo, bruto, mentiroso y por supuesto pagano" (Jacoby, 2004, p.36) ¿Qué generó tal odio y condena? Paine fue crítico de los tres grandes monoteísmos, a pesar de nunca haber rechazado explícitamente la existencia de una deidad. Sus dos grandes pecados fueron poner el bienestar de todas las personas por encima de la lealtad nacional y rechazar las religiones formales de los Cristianos, Judíos y Musulmanes.

Incluso hoy en día, puede resultar difícil para los profesores mencionar que varios de los fundadores de los Estados Unidos hayan sido deístas o secularistas.

Jefferson y Madison fueron secularistas; se puede afirmar con casi total seguridad que Washington, Franklin y John Addams eran deístas. Dado que el cosmopolitismo y el laicismo han estado tan íntimamente relacionados, al criticar uno es probable que el otro también sufra. El actual resurgimiento de las visiones religiosas conservadoras, pueden por lo tanto representar un obstáculo en una discusión justa del cosmopolitismo. 
El patriotismo, no obstante, es la mayor fuerza disuasiva para aceptar el cosmopolitismo. Cuando hablo de aceptación no estoy sugiriendo que tratemos de convertir a los estudiantes al cosmopolitismo por medio de nuestra enseñanza. Sugiero más bien que enseñemos para comprender de manera justa las visiones cosmopolitas y valorarlas sobre la base de dicha comprensión. El cosmopolitismo en sí requiere que sus adherentes escuchen a otros y traten de entender su intención y por lo que están pasando. El patriotismo sin cuestionamientos dificulta, e incluso hace que sea moralmente cuestionable, el compromiso con dicho escuchar.

El patriotismo involucra orgullo por la identidad nacional y dedicación a la preservación y mejoramiento de esa identidad. Siempre se ha esperado que nuestras escuelas inculquen y aumenten el espíritu de patriotismo en nuestros niños y, como se mencionó anteriormente, la cultura promueve el patriotismo a través de una variedad impresionante de celebraciones. Como resultado de esto, el patriotismo tiene un poderoso efecto emocional sobre nosotros. Se nos conmueve por medio de las hazañas de patriotas heroicos y por el llamado a la solidaridad en tiempos difíciles.

En contraste con lo anterior, el cosmopolitismo no parece tener un gran impacto emocional sobre nosotros. Benjamin Barber (1996) se refiere a la "delgadez" del concepto. Simplemente no nos invita a participar con pasión. "Nuestros lazos," escribe, "comienzan de manera provinciana y solo después se expanden" (Barber, 1996, p.34). Pero sin una motivación reflexiva, puede que nunca se expandan, e incluso cuando lo hagan, puede ser que un patriotismo excesivamente ferviente los lleve de vuelta. El patriotismo exacerbado hasta el frenesí, puede producir expresiones de odio difíciles de imaginar incluso años más tarde. Los estadounidenses que eran niños durante la II Guerra Mundial contemplan incrédulos el odio que se nos enseñó a sentir hacia los japoneses.

¿Existe una manera de ejercer algún control sobre el patriotismo de modo tal que no nos abrume y nos proporcione confort para blindar el odio o el prejuicio? Barber concluye,

"La pregunta no es cómo prescindir del patriotismo y el nacionalismo sino cómo convertirlos en algo seguro" (1996, p.36). Jean Bethke Elshtain (1987) está de acuerdo con esta opinión y recomienda un giro hacia lo que ella denomina patriotismo "moderado." Destaca que un patriotismo reflexivo, moderado, debiera inducir un profundo interés por el carácter y bondad de nuestro país - no simplemente orgullo por sus éxitos.

En el espíritu de este patriotismo moderado debemos admitir que algunas veces nuestra nación ha cometido actos dignos de lamentar, y debemos educar a nuestros niños no solo acerca de estos actos sino también acerca de los fuertes desacuerdos que les han seguido. El incidente del Enola Gay es un ejemplo importante. El Enola Gay era el avión donde se transportaba la bomba atómica que destruyó Hiroshima en la II Guerra Mundial. La propuesta de exhibir el avión y su misión en el Smithsonian Museum habría atraído la atención hacia el bombardeo de 1945 y a todo el horror de ese evento. Si bien el bombardeo había ocurrido cincuenta años antes de la exhibición planificada, todavía se podía sentir una gran susceptibilidad, y las objeciones a cualquier recordatorio gráfico de lo que "nosotros" le hicimos a Hiroshima eran 
tan potentes que se decidió simplemente exhibir el avión sin ninguna referencia a su misión. La exhibición planificada fue criticada como anti-estadounidense, sugiriendo que el bombardeo era hasta cierto punto moralmente cuestionable. Guiados por un patriotismo moderado, podríamos encontrar una manera de contarles a nuestros niños no solamente lo que ocurrió sino cómo nuestros recuerdos y explicaciones difieren. Las divergencias de opinión y argumentos suceden antes así como después de las decisiones cruciales. Michael Bess (2006) señala dos propósitos muy distintos en pugna con respecto a la exhibición propuesta del Enola Gay-la conmemoración del fin de una guerra horrible que costó las vidas de tantos soldados aliados que debían ser honrados, y un arrepentimiento moral acerca de cómo se había terminado la guerra. Si bien Bess sugiere que los dos propósitos deben mantenerse separados, se muestra ambivalente al respecto. Dicha separación podría prevenir la clase de clamor inducido por la exhibición propuesta, pero el clamor en sí es algo acerca de lo cual los estudiantes necesitan oír hablar ¿Por qué los sentimientos permanecen de manera tan potente después de cincuenta años? ¿Por qué no podemos aceptar el choque de recuerdos y sentimientos como una lección en sí misma?

Existen muchos temas susceptibles de ser examinados críticamente sólo a partir de esta historia ¿Se deben mantener las ceremonias y exhibiciones memoriales estrictamente ajenas a cualquier tipo de crítica? ¿Contribuye la insistencia en lo sagrado de los memoriales a una prolongación de la guerra? Para los propósitos actuales, sin embargo, en este relato lo importante es notar que a esos ciudadanos que querían narrar la historia completa de Hiroshima, se les acusó de ser anti-estadounidenses, no patriotas. Es casi imposible considerar el patriotismo moderado de Elshtain durante la guerra, y hacerlo años después, cuando los recuerdos de la guerra se despiertan, continúa siendo muy difícil.

En ausencia de la guerra, las escuelas podrían ser capaces de acercar a sus estudiantes a considerar el cosmopolitismo. Martha Nussbaum (1996) ofrece cuatro argumentos para enfocar la educación cívica en el cosmopolitismo. Primero, "a través de la educación cosmopolita, aprendemos más acerca de nosotros mismos” (p.11). Sin el estudio de lo que es ser padres y las prácticas de la crianza en otras partes del mundo, sugiere Nussbaum, es probable que asumamos que nuestras formas son " normales' y 'naturales' para todos los humanos” (1996, p.12). Hago una pausa aquí para señalar que nuestras escuelas no enseñan nada acerca de ser padres y la crianza, y ese descuido probablemente agrava la preocupación de Nussbaum. Nuestros estudiantes se encuentran mal preparados para considerar otras prácticas debido a que no han oído casi ninguna crítica a nuestros métodos de ser padres. A no ser que se enseñe cuidadosamente y con cierta profundidad las maneras de otros, es probable que la conclusión sea que nuestra manera es superior a la de otros, y esta conclusión bien puede apoyar la actual declaración del excepcionalismo estadounidense. Con esa afirmación aceptada ampliamente, convertir al resto del mundo a nuestras maneras se constituye en un deber.

Segundo, a través de una educación cosmopolita, escribe Nussbaum, "avanzamos en resolver problemas que requieren de una cooperación internacional” (p.12). Con un énfasis en el escuchar abogado por Marco Aurelio previamente en su discusión, 
bien podríamos avanzar un poco. El escuchar receptivo es también una característica básica de la ética del cuidado (Noddings, 1984, 2006). Esta clase de escucha es genuinamente abierta; no comienza con una actitud enjuiciadora ni evaluativa. Busca aprender lo que el otro siente y necesita. Anima al oyente a dejarse conmover por la empatía (Slote, 2007).

En tercer lugar, continúa Nussbaum, "reconocemos las obligaciones morales con el resto del mundo que son reales y que de otra manera no se reconocerían" (p.12). Nussbaum plantea un argumento especialmente importante cuando observa que la universalización de los altos estándares de vida de Estados Unidos probablemente conduciría al desastre ecológico. La consideración del consumismo derrochador y la producción de desechos en los Estados Unidos, es un punto central en mi argumento a favor del cosmopolitismo ecológico (Noddings, 2012). Esta idea se discutirá en mayor detalle más adelante.

El cuarto punto de Nussbaum plantea que a través de una educación cosmopolita, "argumentamos de manera consistente y coherente basados en las distinciones que estamos preparados para defender" (p.14). A Nussbaum le preocupa que los "valores compartidos" ensalzados por Richard Rorty y Sheldon Hackney parecieran estar confinados a nuestros límites nacionales ¿Por qué, se pregunta, "deberían estos valores, que nos enseñan a darnos las manos a través de los límites de la etnia, clase, género y raza, perder fuerza cuando llegan a las fronteras de una nación?” (p.14). En respuesta, tenemos que preguntarnos exactamente qué valores hemos seleccionado y por qué Rorty y Hackney piensan que deberíamos “darnos las manos.” La respuesta patriótica tradicional a esto es que queremos preservar la naturaleza político/social distintiva de Estados Unidos y la devoción de sus ciudadanos. Hoy en día, a menudo se elabora la respuesta en términos de mantener al estadounidense al la cabeza del mundo económica y militarmente. Cuando el excepcionalismo estadounidense constituye el valor básico subyacente a todos los demás, entonces muchos otros valores sí se detienen en nuestras fronteras nacionales.

$\mathrm{Si}$ es que vamos a tener alguna oportunidad de fomentar las actitudes cosmopolitas, pienso que debemos comenzar en algún dominio distinto al político. Una posibilidad alentadora es concentrarse en la ecología, en la salud de la Tierra en sí. El bienestar de cada nación depende en último término de la salud de la tierra en la cual todos vivimos. Abordar el cosmopolitismo ecológico en nuestras escuelas requerirá que nuestros docentes crucen las fronteras disciplinares logrando que las conexiones sean centrales al pensamiento ecológico.

\section{Hacia un Cosmopolitismo Ecológico}

Concebimos a nuestro país tanto como hogar y como nación. En los últimos tres siglos, y especialmente en el siglo veinte, la atención de los estadounidenses se ha centrado en la nación, un conjunto de personas con valores cívicos distintivos y una forma particular de gobierno. De modo similar, a nivel global, hemos tendido a concebir el mundo como un conjunto de naciones. A medida que nos movemos hacia el cosmopolitismo ecológico, podemos experimentar un retorno a nuestras raíces y 
comenzar a concentrarnos en la Tierra como una comunidad natural y en los países como hogares interconectados. Esto no implica que debiéramos ignorar las políticas y economías internacionales, pero sí sugiere un giro en el énfasis.

Dicho giro no requiere que creamos en el calentamiento global y en el rol que tienen los seres humanos en causarlo, pero sí requiere que tomemos esas advertencias seriamente. Si el calentamiento global todavía no constituye un hecho científicamente probado (a pesar que muchos de nosotros creemos que sí lo es), toda la gente sensata debe considerarlo como un desastre probable que de ser posible, debiera prevenirse. Comenzamos nuestros estudios y conversaciones globales con el fin de proteger la salud de la Tierra. Este es un interés común por excelencia.

En nuestras escuelas, el giro en el énfasis induciría un cambio correspondiente en el currículo, que se concentraría más en geografía e historia natural, que en historia política. Una vez más, esto no significa que debemos abandonar la historia política. El cambio de énfasis requerirá una coordinación saludable entre las ciencias sociales y las ciencias naturales, enriqueciendo ambas áreas de estudio y dándoles mayor sentido.

Un tercer giro en el énfasis nos llevaría desde una concentración en la vida silvestre (separar las residencias humanas y la naturaleza) hacia una concentración en la ecología. La atención del siglo veinte a la preservación de la vida silvestre fue admirable en muchas formas y, muy justificadamente, los estadounidenses aprecian nuestros grandes parques nacionales. Sin embargo, el foco sobre la preservación de la vida silvestre estaba en satisfacer las necesidades humanas y los parques eran diseñados y mantenidos para promover el deseo de la gente de disfrutar de la vida silvestre. Los parques pasaron a ser áreas de recreo (Steinberg, 2002). Sin desdeñar la importancia que ha tenido el movimiento por la vida silvestre, presenta limitantes obvias; concentra la protección de la naturaleza dentro de áreas de vida silvestre designadas, y dice poco acerca de la condición total de las comunidades naturales. El giro hacia un enfoque ecológico comenzó a tomar fuerza con la publicación de Silent Spring, de Rachel Carson en 1962. Ted Steinberg escribe:

A pesar del uso indiscriminado de la palabra en su libro, Carson contribuyó a transformar ecología en la consigna del movimiento ambientalista. Al contrario de la vida silvestre, concebida como un mundo aparte, la palabra ecología sugería en un sentido, lo opuesto - que toda forma de vida estaba vinculada a una intrincada red interconectada. De esta manera, ella creía que los seres humanos formaban parte del equilibrio de la naturaleza, que no estaban divorciados de ésta en la manera en que algunos defensores de la vida silvestre daban a entender (Steinberg, 2002, p.247).

El énfasis en la ecología ofrece diversos beneficios. En primer lugar, nos entrega un centro de interés global común. En segundo lugar, requiere situarse más allá de la fe en la administración burocrática. La "burocracia" se ha convertido en un marcado término peyorativo, asociado a trámites y toda clase de congestión administrativa, pero inicialmente describía un sistema que ajustaba problemas y soluciones cuidadosamente. La idea era identificar un problema y derivarlo a una agencia designada 
para encontrar una solución eficiente. Además de los "rodeos" por los que el sistema es tan ampliamente criticado, a menudo el pensamiento burocrático llevaba a una proliferación de los problemas generada por la "solución" de un problema inicialmente identificado y asignado a una agencia. Por ejemplo, como señaló Carson, rociar los árboles frutales con pesticidas para preservar la fruta, ocasionaba la desaparición de la provisión de alimentos para los pájaros, y lo que era peor, el envenenamiento a causa del alimento contaminado - de ahí en consecuencia una primavera en silencio sin el canto de las aves. Todavía más, al haber menos pájaros-quiénes controlan las plagas de manera natural — se requerirían incluso más pesticidas. Se ha hecho aparente que el pensamiento burocrático, tanto en los dominios ambientales como sociales, debe supeditarse a un enfoque más holístico, a un reconocimiento de la interdependencia.

Un enfoque ecológico hacia los temas ambientales, también debería tener un efecto positivo en los problemas sociales. En vez de buscar una causa del problema de las escuelas que fracasan, por ejemplo, nos estamos empezando a dar cuenta que enfrentamos no tan solo una causa que es la raíz de los problemas, sino que toda una maraña de causas relacionadas. Gradualmente, estamos comenzando a mirar nuestros problemas acerca de la educación desde una perspectiva ecológica, es decir, con atención a toda la comunidad y a todas sus partes. Tenemos que trabajar de inmediato en diversos frentes, con especial atención a lo que se está haciendo en cada uno. En las escuelas, los alumnos deberían tener oportunidades para estudiar problemas ambientales, tanto en entornos terrestres como oceánicos. Siguiendo con este énfasis en el pensamiento ecológico, los científicos marinos han establecido la importancia de la diversidad (Safina, 2011). Al estudiar el deterioro de los arrecifes de coral en el Caribe, encontraron que un importante crecimiento de alga marina bloqueaba la luz solar necesaria para que los corales bebé vivieran y crecieran ¿Por qué el alga creció fuera de control en el Caribe, pero no así en los arrecifes alrededor de Palaos, en el Pacífico? La respuesta es casi con certeza que la gran población de peces comedores de alga se conservó en Palaos pero se agotó en Belice debido a la pesca comercial. Safina comenta:

Los peces son el motivo por el cuál los corales bebé se han asentado y desarrollado. Debido a que Palaos decidió no autorizar la exportación de sus peces - y ya que su población humana es pequeña comparada con sus vastos arrecifes - ahora tiene: peces, barcos llenos de turistas con dinero para gastar y la tasa de recuperación del coral más alta jamás registrada (2011, p.285).

A medida que los estudiantes leen acerca de la dramática recuperación de los arrecifes de Palaos y la importancia de mirar a toda la comunidad biológica, pueden comenzar a preocuparse acerca de los "avalanchas" de turistas ¿Cómo llegaron los turistas ahí? ¿Cuánta polución del aire es causada por los aviones? ¿Cómo afectan las emisiones de carbono al calentamiento global? ¿Qué tiene que ver el calentamiento global con el aumento en el nivel del mar y la creciente posibilidad que se inunden las islas de Palaos? ¿Cómo preservamos estos lugares maravillosos y naturales sin privar a sus 
poblaciones humanas de oportunidades para alcanzar mayor prosperidad?

El pensamiento ecológico enfatiza el equilibrio y recomienda la diversidad. En la agricultura, así como en la formación de arrecifes, la conservación de la diversidad y el equilibrio es crucial. Los desastres a causa de un monocultivo tales como la hambruna irlandesa de la patata a mediados de 1840 , hoy en día se pueden a menudo evitar por medio de aplicaciones masivas de pesticidas, pero una diversidad de plantas e insectos podría resultar eficaz y mucho menos peligrosa. Al comentar sobre la práctica del monocultivo, Eric Grissell (2001) escribe:

... un único tipo de planta se cultiva en filas controladas a distancias específicas en suelo químicamente alterado, el que es desmalezado con medios mecánicos y químicos. El resultado es que ni siquiera dos plantas pueden interactuar mucho. Hay poca o ninguna diversidad en un sistema como ése. Además, hay cientos o miles de acres bajo esa constante supervisión y bajo esa constante descarga de insecticidas, acaricidas, fungicidas y herbicidas... no hay un equilibrio ecológico o natural en funcionamiento (2001, p. 227).

¿Cómo afecta a la estabilidad la diversidad de especies y hábitats? ¿Cómo puede fomentarse una variedad de insectos, y cómo podríamos utilizarlos para manejar nuestros jardines naturalmente?

El pensamiento ecológico también ha comenzado a ejercer mayor influencia sobre la planificación urbana. En vez de muros vacíos para separar a las personas, ahora los planificadores consideran cómo utilizar el espacio entre los edificios para acercar a las personas. Al escribir acerca de los espacios entre los edificios, Larry Ford comenta:

Buscamos algún sentido de comunidad, un sentido de lugar, un sentido de pertenencia, un sentido de historia o un sentimiento de compromiso y participación (2000, p. 208).

Al considerarlos ecológicamente, los pasajes y los espacios debieran conectarse, no crear barreras y separación.

De manera similar, el enfoque ecológico está reduciendo la separación notoria entre la ciudad y el campo. Muchas ciudades han creado franjas verdes y senderos entre los rascacielos y las líneas ferroviarias y hemos descubierto que los porches y las ventanas bien mantenidos y con buena iluminación reducen la incidencia delictiva, tal vez contribuyendo al sentido de estar juntos en una comunidad. Algunos podrían decir que las ventanas delanteras iluminadas conllevan el sentido que alguien está mirando y es este sentimiento de estar siendo observado el que ha ayudado a reducir la tasa de delitos en algunos vecindarios, pero es igualmente plausible argumentar que esta apertura aumenta nuestro sentido de comunidad y el deseo de mantener su seguridad. A pesar de que el pensamiento ecológico es ahora popular en la planificación urbana, todavía existe controversia (Christensen, McDonald, \& Denning, 2012). Algunos pensadores ecológicos quieren que las ciudades dejen espacio para parques, árboles, pequeños patios y hábitats para pequeños animales, pájaros e insectos. Otros 
(urbanistas) insisten que el simple hecho de concentrar la vida humana en las ciudades mejorará todo el ambiente al reducir el tráfico automovilístico y otras fuentes de emisiones.

La concentración de la vida residencial en las ciudades también reduciría el daño causado al mantener grandes zomas verdes suburbanas con el consiguiente uso masivo de agua y fertilizantes. La esperanza es que los urbanistas y nuevos ecologistas continúen conversando y trabajando conjuntamente para crear ambientes urbanos que sean eficientes, hermosos y que apoyen más la salud. Aquí las palabras claves son, así como lo son en la ecología en sí, conexión, interdependencia y sostenibilidad.

En un nivel global, a los alumnos se les debería crear conciencia de las "zonas problemáticas" de la Tierra - áreas en donde existe la mayor amenaza de extinción de especies. E. O. Wilson señala que:

Estas veinticinco zonas problemáticas ocupan solamente alrededor del 1,4 por ciento de la superficie terrestre del mundo. No obstante, sorprendentemente, son los hogares exclusivos del 44 por ciento de las especies vegetales del mundo y de más de un tercio de todas las especies de aves, mamíferos, reptiles y anfibios. Prácticamente todos se encuentran en serio riesgo de extinción (Wilson, 2002, p.61).

Esto significa que al no tener éxito en proteger estos lugares, la Tierra perdería una variedad increíble de formas de vida. También significa que debido a que el área es relativamente pequeña, preservarla debiera ser una tarea manejable. Sin embargo, debido a que estos lugares ya se encuentran demasiado seriamente dañados y en la mayoría de los casos, demasiado densamente poblados, la tarea en realidad será difícil.

Piénsese cuán enriquecidas y relevantes podrían llegar a ser la clase de geografía ¿Pueden los alumnos localizar el bosque del Amazonas, las Antillas Mayores, el bosque del Congo, los Ghats Occidentales de la India, Filipinas y Nueva Caledonia? ¿Pueden nombrar unas pocas especies en riesgo en estas áreas? ¿Qué más está bajo amenaza cuando se reducen o destruyen antiguos bosques? Pero las clases no debieran limitarse a las zonas problemáticas terrestres. Recuerden la mención a los arrecifes de coral en deterioro ¿Dónde están los arrecifes muriendo y dónde están prosperando?

Conviene advertir, además, que la concentración en la ecología conduce necesariamente a lo que podría llamarse ecología disciplinar, es decir, los educadores tendrán que considerar cómo las asignaturas que se enseñan ahora en nuestras escuelas se conectan entre ellas y con la vida misma. Ya no podemos seguir apoyando un sistema que separa cada asignatura escolar de todas las otras. Además de que les vaya bien en una prueba, ¿qué sentido tiene que aprendan un conjunto interminable de hechos y habilidades inconexas? (Noddings, 2007, 2012).

El estudio de la ecología global debería ser fascinante, pero también puede inducir a un sentimiento de indefensión en alumnos de nivel pre-universitario ¿Qué pueden hacer ellos acerca de esos tremendos problemas? Para responder esta pregunta, deberíamos considerar la ecología local y cómo los estudiantes pueden participar de manera útil en proyectos ambientales valiosos. 


\section{Un Retorno Ecológico a Nuestras Raíces}

Debido a que a tantos niños no se les permite salir al aire libre y moverse a sus anchas, puede ser incluso más importante para las escuelas motivar a los alumnos a estar en contacto con la naturaleza y, con ese fin, con objetos que necesiten repararse y con material que pueda utilizarse para crear cosas. Wendell Berry ha comentado:

Creo que por muchas razones — políticas, ecológicas y económicas — las más grandes inteligencias y talentos deberían estar en todos los hogares y trabajos del país. Y por lo tanto, lo que deseo para nuestras escuelas es lo opuesto a lo que desean nuestros actuales partidos políticos y actuales políticas en educación y cultura. Wes Jackson ha argumentado que para equilibrar o reemplazar su actual, única especialidad en movilidad ascendente, nuestras escuelas debieran ofrecer una especialidad en un retorno a nuestras raíces (Berry, 1995, p. xi).

El pensamiento ecológico sobre nuestro ambiente comienza en nuestros propios patios. Después de discutir acerca de los problemas de nuestros grandes ríos, Sara Stein nos aconseja dar una mirada en mayor detalle:

Pero miremos algo más pequeño que el Colorado. Miremos las filtraciones, riachuelos, pozos, cuencas, ciénagas, diques, piletas. Examinemos la faz del continente a través de una lupa, poniendo menos atención a las grandes protuberancias y declives y más atención a la fina textura de su piel, sus poros y pequeñas arrugas. Vamos a los detalles minuciosos de las pozas. Miremos nuestros propios patios y preguntémonos dónde pueden beber las mariposas (Stein, 1993, pp.175-176).

Estamos defraudando a nuestros niños al separarlos tan tempranamente y de manera tan definitiva del trabajo manual y con la naturaleza. Al hacerlo también los estamos separando de otros seres humanos que trabajan con sus manos y de una parte importante de ellos mismos. Matthew Crawford cita al director de la Asociación de Profesores Agrícolas de California quien dice: "Tenemos una generación de estudiantes que puede responder preguntas en pruebas estandarizadas, que conoce datos pero que no puede hacer nada" (Crawford 2009, p.12). Esto no solamente es contraproducente para el tipo de trabajo esencial en el cosmopolitismo ecológico, sino que además menoscaba el desarrollo de todos los seres humanos. Los proyectos prácticos del mundo real nos conectan entre nosotros y con una parte esencial de nuestro ser. Cuando Kieran Egan recomienda los proyectos como una parte del aprendizaje en profundidad, señala que:

Solamente quiero agregar la observación relacionada con nuestra ignorancia catastrófica del mundo natural, y sus consecuencias cognitivas, como otra razón para considerar el valor educativo del AeP [aprendizaje en profundidad]. En especial si escogemos nuestros temas desde el mundo natural, podemos capacitar 
a cada estudiante para que acumule una cantidad, así como una intensidad significativa de conocimiento, que podrían, de alguna manera, encaminarse a que nos libráramos de nuestra actual incapacidad para pensar bien acerca del mundo natural y de nuestro lugar en él (Egan, 2010, pp.17-18).

La educación escolar no debiera separarnos de las dimensiones físicas y emocionales de nuestras vidas. De hecho, la palabra clave en ecología es conexión- no solo entre las formas de vida en la Tierra sino también entre los temas que estudiamos, los objetos que utilizamos, las condiciones físicas en las que vivimos, nuestras casas y jardines, cómo y por qué nos desplazamos de un lugar a otro. Esta observación sugiere que en las escuelas podríamos tener una reducción rentable del énfasis en la historia política y prestar más atención a la historia de los hogares, de ser padres y de la infancia. No es necesario sufrir una pérdida intelectual al hacer este cambio, ya que-como Dewey destacaba años atrás - el poder intelectual de una asignatura no se determina por su título sino por la forma en que se estudia. El álgebra no es inherentemente más intelectual que el ramo de cocina.

A medida que motivamos a los alumnos a cuidar sus propios patios, mantener sus jardines y apreciar la variedad de formas de vida a su alrededor, también deberíamos ayudarles a comprender que las personas alrededor de todo el mundo sienten cariño por sus propios lugares naturales - dentro y fuera de las ciudades. Cuidar nuestra propia parte de la Tierra debería conducir de manera bastante natural a un deseo por comprender y proteger el mundo más grande, la Tierra en sí.

Hoy en día, la educación debería ofrecer a los estudiantes la seguridad para que pudieran proclamarse como ciudadanos del mundo. El patriotismo fuertemente nacionalista simplemente ya no es saludable ni para la Tierra ni para el país que amamos. Junto con un cambio en el énfasis desde un patriotismo nacional hacia un cosmopolitismo ecológico, se debería invitar a los estudiantes a considerar cómo la música, los uniformes y las celebraciones apoyan el nacionalismo. Pueden contrastarse, por ejemplo, dos canciones patrióticas que todos los escolares estadounidenses aprenden: "The Star-Spangled Banner" y "America the Beautiful." En la primera, cantamos acerca de banderas, cohetes, fortificaciones, lucha, la desolación de la guerra, la valentía y el triunfo. En la segunda, cantamos acerca de cielos espaciosos, olas de grano ámbar, misericordia, autocontrol, el éxito como nobleza y la hermandad desde el mar hasta el mar resplandeciente ¿Describe el contraste una diferencia acerca de cómo podríamos expresar nuestro amor por el país? ¿Supongan que el Día del Árbol (Arbor Day) se celebrara con el entusiasmo del Día de la Independencia?

Finalmente, debemos retomar una extensión del segundo beneficio de enseñar cosmopolitismo de Nussbaum - avanzar en la resolución de problemas que requieren de una cooperación internacional. El compromiso con un cosmopolitismo ecológico puede servir como un acompañamiento o, a menudo, como preludio del cosmopolitismo político. A medida que trabajamos para resolver los problemas ambientales, nos vamos conociendo mejor. Aprendemos a ejercer el requisito principal del cuidado - escuchar. Al trabajar juntos, escuchando, comenzamos a preocuparnos no solamente por los problemas y su solución. Comenzamos a interesarnos por 
aquellos con los que trabajamos; herirlos o destruirlos no es una opción. Es en esta etapa donde podemos abordar de manera segura nuestras diferencias políticas.

\section{Referencias}

Barber, B. (1996). Constitutional faith. En M.C. Nussbaum, For Love of country? Ed.

J.Cohen (pp. 30-37). Boston: Beacon Press.

Berry, W. (1995). Another turn of the crank. Washington, D.C.: Counterpoint.

Bess, M. (2006). Moral dimensions of World War II. New York: Alfred A. Knopf.

Carson, R. (1962). Silent spring. Boston: Houghton Mifflin.

Christensen, J., McDonald, R., \& Denning, C. (2012). Ecological urbanism for the $21^{\text {st }}$ Century. The Chronicle Review, Jan. 27, B9-B11.

Crawford, M.B. (2009). Shop class as soulcraft. New York: Penguin Press.

Egan, K. (2010). Learning in depth. Chicago: University of Chicago Press.

Elshtain, J.B. (1987). Women and war. New York: Basic Books.

Ford, L.R. (2000). The Spaces between buildings. Baltimore: Johns Hopkins University Press.

Grissell, E. (2001). Insects and gardens: In Pursuit of a garden ecology. Portland, OR: Timber Press.

Jacoby, S. (2004). Freethinkers. NewYork: Metropolitan Books.

Noddings, N. (1984). Caring: A Feminine approach to ethics and moral education. Berkeley: University of California Press.

Noddings, N. (2006). Critical lessons: What our schools should teach. Cambridge: Cambridge University Press.

Noddings, N. (2007). When school reform goes wrong. New York: Teachers College Press.

Noddings, N. (2012). Peace education: How we come to love and hate war. Cambridge: Cambridge University Press.

Nussbaum, M.C. (1996). For Love of country? Ed. J. Cohen. Boston: Beacon Press.

Safina, C. (2011). The View from lazy point. New York: Henry Holt.

Slote, M. (2007). The Ethics of care and empathy. New York: Routledge.

Stein, S. (1993). Noah's garden: Restoring the ecology of our own back yards. Boston: Houghton Mifflin.

Steinberg, T. (2002). Down to earth. Oxford: Oxford University Press.

True, M. (1995). An Energy field more intense than war. Syracuse: Syracuse University Press.

Wilson, E.O. (2002). The Future of life. New York: Alfred A. Knopf. 